\title{
Bio- nanomaterials for versatile bio- molecules detection technology
}

\author{
Ravindra P. Singh ${ }^{1^{*}}$ and Jeong-Woo Choi $^{1,2 *}$ \\ ${ }^{1}$ Interdisciplinary program of Integrated Biotechnology, Sogang University, 1 Sinsoo-Dong, Mapo-Gu, \\ Seoul 121-742, South Korea \\ ${ }^{2}$ Department of Chemical and Biomolecular Engineering, Sogang University, 1 Sinsoo-Dong, Mapo-Gu, \\ Seoul 121-742, South Korea
}

"Corresponding author. Tel: (+82) 2-705-8480; Fax: (+82) 2-3273-0331; E-mail: rpsnp169@gmail.com (R. P. Singh), jwchoi@sogang.ac.kr (J. W. Choi)

Received: 2 April 2010, Revised: 24 April 2010 and Accepted: 26 April 2010

\section{Author's Biography}

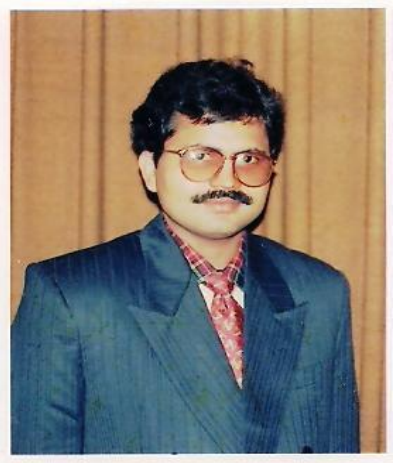

Ravindra P. Singh received the PhD degree in Biochemistry from the Lucknow University, India, in 1999. From 2000 to 2003, he was a postdoctoral fellow at the IGI, France and then at IHBAS, Delhi, India as a senior demonstrator. In 2003 to 2007, he joined the Biosensor group in National Physical Laboratory New Delhi, India as postdoctoral Research Associate and Pool scientist CSIR and then in 2007 joined as Research Professor at Sogang University, South Korea, working on biosensors, biochips, and biomemory. His research interests include all aspects of design, fabrication, and characterization of nanostructures and nanobiosensors, biosensors, biochips, protein chips, biomemory, and lab-on-a-chips.

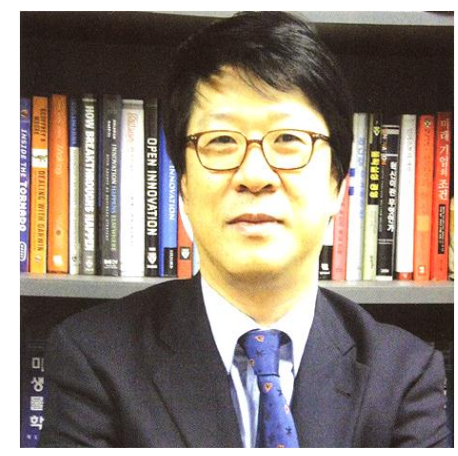

Jeong-Woo Choi is Professor of Department of Chemical and Biomolecular Engineering, and Director of Interdisciplinary Program of Integrated Biotechnology of Sogang University in Korea. He was a president of The Korean Biochip Society in 2009. He received Ph.D. in Department of Chemical \& Biochemical Engineering, Rutgers University, USA (1990), D. Eng. in Department of Biomolecular Engineering, Tokyo Institute of Technology, Japan (2003), and MBA in University of Durham, UK (2007). He was a visiting researcher in IBM Almaden Research Center, USA (1994) and in Mitsubishi Electronics Advanced Technology R\&D Center, Japan (1996). He has done researches on the fields of nanobioelectronics, especially for bio-memory, protein chip, and cell chip. He has published about 300 journal papers in bioelectronics and biotechnology field. Copyright (C) 2010 VBRI press.

\section{Dear Editors,}

Over the past few years, bionanomaterial research has emerged as a new exciting field and the importance of DNA, RNA and peptides in designing bionanomaterials for the fundamental development in biotechnology and nanomaterials has begun to be recognized as a new interdisciplinary frontier in field of life science and material science. Great advances in nanobiochip materials, nanoscale biomimetic materials, nanomotors, nanocomposite materials, interface biomaterials, and nanobiosensor and nano drug delivery systems have enormous prospect in industrial, defense, and clinical medicine applications. Bio-molecules are very important elements in nanoscience and nanotechnology. Peptide nucleic acids (PNAs) replace DNA as a probe for biomolecular tool in the molecular genetics diagnostics, cytogenetic, and various pharmaceutical potentials as well as for the development of sensors/arrays/chips and many more purposes. One of the current aspects related with PNAs is the making of a new hot device for the commercial applications e.g. nanobiosensor arrays [1]. 
The integration of nanotechnology, microfabrication techniques, and miniaturized devices with novel biochemical detection methodologies, leads to very sensitive and fast assays for the detection of desired biomolecules related with various commercial sectors. Nanotechnology involves the assembly of small molecules into complex architectures for an improved function by controlling the precise location of each atom in a 3dimensional space. It form larger functional elements and is being explored as potential way to fabricate nanometer-size devices [2]. Numerous reports are documented regarding the use of oligonucleotides for building nanostructures, which include DNA matrices based on subunits of fixed Holliday junctions, streptavidin-DNA fragment nanoparticle networks, DNA dendrimer formations for drug delivery, molecular tweezers (ssDNA based) and molecular switches [3-4]. PNAs are promising connector for the assembly of DNA-based nanostructures with an exceptional ability to hybridize to sequences within the duplex DNA by strand invasion. High affinity binding by PNA has already been used for nanostructure assembly, with applications for labeling of DNA and strand invasion into DNA hairpins and tetra loop motifs. The bis-PNAs are two PNA sequences with a tethering spacer region. Amino acids were included in some of the spacer regions to increase the distance between the PNA sequences. The ability of bisPNAs to assemble DNA with simple chemical modifications could be used in generating DNA: bis-PNA: DNA units for nanotechnology and DNA nanostructure assembly [5-6].

Biosensors are an emerging area of interdisciplinary research. Different types of biosensors are used with their advantages and limitations with a different transducer forming the biosensing devices for the detection of various kinds of desired bio-molecules. Nucleic acid elements including aptamers, DNAzymes, aptazymes, and PNA are widely used in nanobiotechnology (lab-on-a-chip, nanobiosensors array) $[\mathbf{1 , 7 ]}$. In addition, the nano biosensor can be easily integrated into disposable polymer lab-on-achips for numerous applications in biochemical analysis and clinical diagnostics. The recent developments in nanobiosensors and their applications in biology especially in medical diagnostics and the concept of coordinated nanobiosensors integrate desirable properties of the individual components: protein machinery for sensitivity and specificity of binding, peptide or nucleic acid chemistry for aligning the various electron-transducing units and the nanoelectrodes for enhancing sensitivity in electronic detection. Results from these systems focus the potential advantages of using nanoscale biosensors area, which will change biomedical diagnostics and treatments drastically. The development and application of nanodevices in biology and medicine will have enormous implications for society and human health [8].

The biosensors integrated with new technologies in molecular biology, microfluidics, and nanomaterials have applications in agricultural production, food processing, clinical care and environmental monitoring for rapid, specific, sensitive, inexpensive, in-field, on-line and/or realtime detection of pesticides, antibiotics, pathogens, toxins, proteins, microbes, plants, animals, foods, soil, air, and water. Thus, ulta-sensitive biosensors are excellent analytical tools for pollution monitoring by which working towards implementing the legislation to safeguard our biosphere is possible. The future emerging trends towards biosensor development in the context of bioelectronics, nanotechnology, miniaturization, and especially biotechnology seem to be all growing areas that will have a remarkable influence on the development of new biosensing platform to resolve our future clinical diagnostics and severe pollution problems concerning not only human health but also all living entities. To understand biological processes at a single molecule level and to implement them for the possible future applications in nanobiotechnology is of current interest. Single molecule studies enabled probe have opened exciting avenues of research, especially in nanoscience and nanotechnology for the versatile bio-molecule detection technology [9].

\section{With warm regards}

Ravindra P. Singh, $\mathrm{PhD}$

Jeong-Woo Choi, $\mathrm{PhD}$

\section{References}

1. Singh, R.P.; Oh, B.K.; Koo, K.K.; Jyong, J.Y.; Jeong, S.; Choi, J.W. Biochip. J. 2008, 2, 223.

2. Rothemund, P.W.K. Nature 2006, 440, 297.

3. Yurke, B.; Turberfield, A.J.; Mills A.P.J.; Simmel, F.C.; Neumann, J.L. Nature 2000, 406, 605.

4. Park, S.J.; Taton, T.A.; Mirkin, C.A., Science 2002, 295, 1503.

5. Singh, R.P. Oh, B.K., Choi, J.W. 2010, doi:10.1016/j.bioelechem.2010.02.004.

6. Seeman, N.C. Annu. Rev. Biophys. Biomol. Struct. 1998, $27,225$.

7. Tombelli, S.; Minunni, M.; Mascini, M., Biosens. Bioelectron. 2005, 20, 2424.

8. Seeman, N.C. Trends Biotech. 1999, 17, 437.

9. Jianrong, C.; Yuqing, M.; Nongyue, H.; Xiaohua, W.; Sijiao, L. Biotechnol. Adv. 2004, 22, 505. 